\title{
LA COMPRENSIÓN DE LA PERSUASIÓN EN NIÑOS Y NIÑAS DE 6 A 12 AÑOS
}

\author{
María José Linero \\ Universidad de Málaga \\ mjlinero@uma.es \\ Carmen Barajas \\ Universidad de Málaga \\ María Mayoral \\ Universidad de Málaga
}

Recepción Artículo: 17 mayo 2021

Admisión Evaluación: 17 mayo 2021

Informe Evaluador 1: 19 mayo 2021

Informe Evaluador 2: 25 mayo 2021

Aprobación Publicación: 01 junio 2021

\section{RESUMEN}

El presente trabajo tiene como objetivo general el estudio de cómo los niños/as comprenden el acto comunicativo de persuasión (aquel en el que se interpreta que una persona quiere conseguir algo de otra) y cómo esta comprensión se relaciona con importantes variables del desarrollo psicológico. A partir de este objetivo general se derivan dos objetivos específicos. El primero consiste en analizar la capacidad de comprensión de la persuasión en niños/as de Educación Primaria en función de la realización de inferencias mentalistas acerca de la perspectiva cognitiva del persuasor (su estado mental de creencia, su motivación/actitud, y su intención al intentar persuadir), así como de la realización de inferencias mentalistas acerca de la perspectiva tanto cognitiva como emocional de la persona persuadida (su estado mental de creencia y su estado emocional, ambos causados por las palabras del persuasor). El segundo objetivo específico consiste en examinar cómo estas atribuciones que hacen los niños se relacionan con la habilidad lingüística y la Teoría de la Mente. Para ello, se han administrado a 103 niños/as de 6 a 12 años una tarea de evaluación de la comprensión de una persuasión, el test PPVT-III para obtener el nivel de desarrollo lingüístico infantil, y un conjunto de tareas de evaluación de distintas habilidades mentalistas. Los resultados indican que el nivel de desarrollo lingüístico infantil se relaciona con la inferencia de la intención del persuasor, así como con las referencias explícitas al estado de creencia y emoción que desarrolla la persona persuadida. Por otra parte, Ios resultados también han puesto en evidencia que las habilidades de inferencia mentalista más sofisticadas están relacionadas con la atribución de la intención al persuasor y con la referencia explícita al estado emocional de la persona persuadida.

Palabras clave: persuasión; lenguaje; teoría de la mente; educación primaria 


\title{
LA COMPRENSIÓN DE LA PERSUASIÓN EN NIÑNOS Y NIÑAS DE 6 A 12 AÑOS
}

\begin{abstract}
Understanding of persuasion in children from $\mathbf{6}$ to 12 years old. The present work has as a general objective the study of how children understand the communicative act of persuasion (one in which it is interpreted that a person wants to get something from another) and how this understanding is related to important variables of psychological development. From this general objective two specific objectives are derived. The first consists of analyzing the ability to understand persuasión in primary school children based on making mentalistic inferences about the persuader's cognitive perspective (their mental state of belief, their motivation/attitude, and their intention trying to persuade), as well as making mentalistic inferences about both the cognitive and emotional perspective of the persuaded person (his mental state of belief and his emotional state, both caused by the words of the persuader). The second specific objective is to examine how these attributions that children make are related to linguistic ability and Theory of mind. For this, 103 children from 6 to 12 years old have been administered an assessment task of the understanding of persuasion, the PPVT-III test to obtain the level of child language development, and a set of assessment tasks of different mentalistic abilities. The results indicate that the child s level of linguistic development is related to the inference of the persuader s intention, as well as to the explicit references to the state of belief and emotion that the persuaded person develops. On the other hand, the results have also shown that the most sophisticated mentalistic inference skills are related to the attribution of the intention to the persuader and to the explicit reference to the emotional state of the persuaded person.
\end{abstract}

Keywords: persuasion; language; theory of mind; primary school

\section{INTRODUCCIÓN}

La persuasión es la habilidad social con la que las personas pretenden conseguir que otros hagan, piensen o sientan lo que ellas desean, por lo que requiere de habilidades sociocognitivas sofisticadas que posibiliten la comprensión de los estados mentales de otros (Bartsch, London y Campbell, 2007). Junto con estas habilidades mentalistas, el uso social de la persuasión requiere la adquisición de unos niveles adecuados de competencia comunicativa y lingüística que permitan la correcta interpretación y producción de los argumentos persuasivos. En definitiva, las habilidades mentalistas y lingüísticas podrían representar elementos fundamentales tanto a la hora de interpretar correctamente los intercambios comunicativos persuasivos como a la de elaborar razonamientos con los que intentar persuadir a otros.

La teoría de la mente puede ser definida como la habilidad para atribuir estados mentales (por ejemplo, intenciones, deseos, y conocimientos) a uno mismo y a los demás al mismo tiempo que se comprende que los otros tienen deseos, actitudes e intenciones diferentes a los nuestros (Premack y Woodruff, 1978). Se trata de una habilidad imprescindible para el desarrollo social, puesto que la atribución de estados mentales nos permite tanto la explicación de la conducta de los demás como la predicción de esta en función de los contenidos mentalistas que les imputamos. Como muchos estudios han demostrado, la gente no nace con esta habilidad, sino que se desarrolla y se vuelve más compleja a lo largo de la infancia y la adolescencia (Miller, 2012).

Los estados mentales de los otros no son directamente observables y los investigadores han diseñado modelos para representar cómo funcionan, así como tareas en las que se propicia su inferencia. Concretamente, la evaluación de la teoría de la mente en los niños se ha realizado habitualmente con tareas planificadas para comprobar si son capaces de atribuir una creencia falsa a un personaje y predecir así su comportamiento. Los niños con un desarrollo típico superan en torno a los cuatro años y medio las tareas clásicas de falsa creencia, mientras que antes de esta edad los niños fracasan porque se dejan Ilevar por su propio estado de conocimiento, y no son capaces de atribuir al personaje un estado mental distinto del suyo propio, por lo que predicen erróneamente su comportamiento (Wimmer y Perner, 1983; Wellman, Cross y Watson, 2001). Estos datos han propiciado que se considere la edad de cuatro años y medio como clave para el desarrollo de las habilidades de teoría de la mente, aunque antes de esa edad se observa que los niños desarrollan capacidades para predecir comportamientos en los demás. 
Wellman y Liu (2004) han propuesto una progresión en la evolución de la teoría de la mente que comienza con la capacidad de atribuir a otros deseos diferentes de los propios, y continúa con la atribución a otros de creencias contrarias a las propias. A continuación, apuntan la comprensión de la necesidad de acceder a la información para poder hacer uso de esta, seguida por la comprensión de que otros pueden albergar creencias falsas sobre la realidad que guían erráticamente su comportamiento. Este último logro, la atribución de creencia falsa al otro 0 de primer orden, ha sido evaluado mediante tareas muy variadas, como por ejemplo la tarea de falsa creencia sobre el contenido o de contenido inesperado de un recipiente, la tarea de falsa creencia por el cambio de localización de un objeto, o la tarea de atribución de una emoción en función de una creencia errónea. Finalmente, Wellman y Liu señalan la comprensión de que una persona puede pretender ocultar sus sentimientos a los demás mostrando sentimientos contrarios a los que realmente tiene y, por tanto, tratando de crear una creencia falsa en Ios otros. Este logro requiere realizar atribuciones de falsa creencia más complejas, las llamadas de segundo orden, y se alcanza habitualmente a partir de los 7 años. En las tareas diseñadas para su evaluación se valora la capacidad del niño para atribuir a una persona una creencia sobre la creencia de otra persona, o una creencia sobre la emoción de otra persona, etc. (Miller, 2012).

El estudio de 0 Hare, Bremner, Nash, Happé y Pettigrew (2009) indica que no es hasta los 9 años cuando los niños describen y se refieren explícitamente a los estados mentales que hay detrás de un comportamiento, como cuando expresaban que "el ladrón pensó que el policía sabía que había robado en la tienda". Esto explicaría que la ejecución de los niños en tareas de comprensión de persuasión y de sarcasmo presente muchas dificultades, dándose el caso de que niños de hasta 12 años proporcionen solo explicaciones parciales de estas situaciones de uso social del lenguaje.

Hay que subrayar que la teoría de la mente no es un constructo monolítico, sino que incluye aspectos tanto cognitivos como emocionales (Lonigro, Baiocco, Baumgartner y Laghi, 2017). Así, la teoría de la mente cognitiva se refiere a la habilidad para realizar inferencias acerca de los estados mentales epistémicos o de conocimiento de las personas (creencias verdaderas, creencias falsas, intenciones, opiniones, conjeturas, etc.), y la teoría de la mente afectiva o emocional implica la inferencia de estados epistémicos o emocionales. La consideración de esta doble naturaleza de la teoría de la mente es interesante, ya que una y otra podrían relacionarse diferenciadamente con la comprensión de determinados aspectos cuando se interpretan los argumentos persuasivos.

Por otra parte, algunos estudios han encontrado que la habilidad lingüística afecta a la ejecución de los niños de edad preescolar en tareas de conocimiento de la persuasión (Donohue, Henke y Donohue, 1980). Asimismo, un número importante de investigadores ha señalado que las habilidades lingüísticas tienden a ser un predictor consistente de la teoría de la mente (Jenkins y Astington, 1996; Lillard y Kavanaugh, 2012; Watson, Painter y Bornstein, 2001). En esta dirección, Milligan, Astington y Dack (2007) indicaron que existe una clara relación entre el desarrollo lingüístico infantil y la adquisición de habilidades de inferencia mentalista, puesto que muchos estudios de su meta-análisis han evidenciado que existe una relación significativa entre cada tipo de habilidad lingüística y la ejecución infantil en tareas de falsa creencia: el lenguaje en general, la semántica, el vocabulario receptivo, la sintaxis y la memoria para las oraciones completivas. El aspecto de vocabulario receptivo ha sido considerado en numerosos trabajos, y generalmente ha sido evaluado mediante pruebas en las que se le pide al niño que señale qué dibujo de entre cuatro representa la palabra que el examinador le está pronunciando. Según Milligan et al. (2007), esto sería debido a que la prueba de vocabulario receptivo proporciona la medida más pura de una habilidad lingüística aislada, puesto que no presenta solapamiento con otras habilidades lingüísticas.

Varios trabajos han señalado que las habilidades de inferencia mentalista predicen la comprensión de la persuasión, concretamente a través de la publicidad. En este sentido, McAlister y Cornwell (2009) encontraron que los niños de edad preescolar con mejor puntuación en tareas de evaluación de teoría de la mente comprendían mejor los anuncios publicitarios (incluso cuando se controlaban la edad y la competencia lingüística). Debido a la corta edad de los niños (cuatro años de media), los investigadores solo examinaron si estos comprendían que los anuncios estaban diseñados para fomentar el consumo del producto, y no contemplaron si los niños diferen- 


\section{LA COMPRENSIÓN DE LA PERSUASIÓN EN NIÑOS Y NIÑAS DE 6 A 12 AÑOS}

ciaban entre mensajes meramente informativos versus persuasivos. Más recientemente, Lapierre (2015) ha estudiado si las habilidades de teoría de la mente en niños de educación primaria ( 6 a 9 años) predecían la comprensión plena de los mensajes publicitarios. Los resultados indican que la detección por parte de los niños de la intención de venta del producto está relacionada con el desarrollo de las habilidades de teoría de la mente, más allá de la influencia de la edad y de la competencia lingüística, y que en general los niños tienen dificultades para comprender íntegramente el acto persuasivo, concretamente a la hora de identificar las intenciones persuasivas genuinas, aquellas que pretenden que se cambie de actitud y de opinión hacia un producto u objeto determinado.

Un estudio de referencia sobre la comprensión de la persuasión es el de Happé (1994), quien la incluyó como un uso no literal del lenguaje en su Batería de Historias Extrañas, un conjunto de relatos cortos que requiere la atribución de estados mentales a otros para ser interpretados adecuadamente. En estas historias se le hacen al niño únicamente dos preguntas: si es verdad lo que dice el personaje (detección de significado no literal del mensaje) y por qué dice eso (identificación de la motivación del personaje). Es decir, en la tarea diseñada por Happé se valora únicamente cómo el niño evaluado infiere el estado mental de uno de los personajes de la historia, el del hablante (pero no el del receptor), y se considera exclusivamente cómo el niño atribuye al hablante una motivación 0 actitud general a la hora de persuadir (pero no su creencia verdadera o su intención persuasiva).

\section{OBJETIVOS DE LA INVESTIGACIÓN}

El presente trabajo tiene como objetivo general el estudio de cómo los niños en etapa escolar comprenden el acto comunicativo de persuasión y cómo esta comprensión se relaciona con las habilidades mentalistas y la competencia lingüística. A partir de este objetivo general se derivan dos objetivos específicos. El primero consiste en analizar la capacidad de comprensión de la persuasión en los niños en función de la realización de inferencias mentalistas acerca de la perspectiva cognitiva del persuasor (su estado mental de creencia, su motivación/actitud, y su intención al intentar persuadir), así como de la realización de inferencias mentalistas acerca de la perspectiva tanto cognitiva como emocional de la persona persuadida (su estado mental de creencia y su estado emocional, ambos causados por las palabras del persuasor). El segundo objetivo específico consiste en examinar cómo estas atribuciones que hacen los niños se relacionan con sus habilidades de teoría de la mente cognitiva y emocional y su nivel de competencia lingüística.

\section{MÉTODO}

\section{Participantes}

El estudio se llevó a cabo con una muestra de 103 niños/as (50 niñas y 53 niños) con una edad comprendida entre los 6 y 12 años $(M=9,66 ; D T=1,82)$, pertenecientes a centros educativos públicos de Educación Primaria.

\section{Instrumentos}

1. Tarea de comprensión de persuasión

(Historia basada en la Historia Extraña de "Persuasión" de Happé, 1994)

Evalúa la habilidad para atribuir al personaje la motivación de causar un estado

emocional (pena) en otro (el cocinero) créandole una idea falsa para así conseguir un beneficio personal.

Historia: Bruno siempre tiene hambre. Hoy en el colegio ponen su comida favorita,

salchichas con patatas. Es un niño muy agonioso y le gustaría que le pusieran más salchichas que a los demás, incluso aunque su madre le hará una comida estupenda cuando llegue a casa. Pero hay solo dos salchichas para cada uno. Cuando le toca a Bruno que le sirvan, le dice al cocinero: " $¡ 0 h$, ¿podría echarme cuatro salchichas?... porque no voy a tener nada de cena cuando llegue a casa!"

Codificación y puntuación

1) Perspectiva cognitiva del hablante

- Creencia (primer orden): ¿Bruno sabe que su madre le va a poner de cenar cuando llegue a casa? 
1 punto si atribuye la creencia (sí que lo sabe)

- Motivación/Actitud: Cuando Bruno le dice al cocinero "..."; ¿Por qué le dice eso?

1 punto si atribuye la motivación para mentir refiriéndose a la consecuencia material (ej. para que le echen más)

2 puntos si atribuye la motivación para mentir refiriéndose explícitamente al estado mental que se pretende (ej. porque quiere que se lo crea)

-Intención (segundo orden): Cuando Bruno le dice al cocinero "....; ¿ Bruno quiere que el cocinero crea que no va a tener nada de cena cuando llegue a casa?

1 punto si hace el razonamiento de segundo orden (sí que quiere)

(2) Perspectiva del oyente

- Cognitiva. Creencia del oyente (segundo orden). Interpretación del efecto epistémico de las palabras del hablante en el oyente: Cuando Bruno le dice al cocinero "..."; ¿El cocinero se va a creer que Bruno no tendrá nada de cena cuando llegue a casa?, ¿Por qué si /no?

1 punto si atribuye implícitamente la perspectiva cognitiva del oyente, de segundo orden (ej. "sí, porque puede que sea un niño pobre y no tiene cena")

2 puntos si atribuye explícitamente la perspectiva cognitiva del oyente, de segundo orden (ej. "sí, porque como nunca ha ido a su casa, no sabe que su madre le pone cena")

- Emocional. Interpretación del efecto emocional de las palabras del hablante en el oyente: Cuando Bruno le dice al cocinero "..."; ¿Tú crees que al cocinero le da pena de Bruno y le pone cuatro salchichas?, ¿Por qué si /no?

1 punto si atribuye implícitamente el efecto emocional en el oyente, de segundo orden (ej. "sí, se las va a poner, porque como no tendrá cena...")

2 puntos si atribuye explícitamente el efecto emocional en el oyente, de segundo orden (ej. "sí, se las va a poner, porque se lo va a creer")

2. Test de Vocabulario en Imágenes Peabody III (PPVT-III) (Dunn, Dunn y Arribas, 2006)

Evalúa el nivel de vocabulario receptivo. Se ha obtenido la puntuación de edad equivalente de comprensión léxica.

3. Tareas de Teoría de la Mente. Se administró una selección de tareas sobre demandas cognitivas diferentes:

- Falsa creencia sobre contenido (Perner, Leekam y Wimmer, 1987). Evalúa la destreza para atribuir una creencia falsa a otro sobre el contenido de un recipiente.

- Codificación y puntuación. 0 puntos: no atribuye falsa creencia; 1 punto: apariencia; 2 puntos: razonamiento implícito (no ha visto lo que hay); 3 puntos: razonamiento mentalista explícito (no sabe que hay X).

- Falsa creencia sobre localización de primer orden (Baron-Cohen, Leslie y Frith, 1985). Mide la capacidad de atribuir una falsa creencia sobre el lugar en el que se encuentra un objeto.

Codificación y puntuación. 0 puntos: no atribuye falsa creencia; 1 punto: Iocalización (porque estaba ahí); 2 puntos: razonamiento implícito (no ha visto el cambio); 2 puntos: razonamiento mentalista explícito (no sabe que la han cambiado).

- Tarea de creencia-emoción positiva (Harris, Johnson, Hutton, Andrews y Cooke, 1989). Valora la competencia para atribuir a otra persona una emoción positiva a partir de una creencia equivocada.

Codificación y puntuación. 0 puntos: no atribuye emoción positiva; 1 punto: referencia al supuesto contenido ("porque le gusta X"); 2 punto: razonamiento implícito ("porque no ha visto que hay Y"); 3 puntos: razonamiento mentalista explícito ("se pondrá contento porque creerá que hay X").

- Tarea de creencia-emoción negativa (Harris et al., 1989). Valora la competencia para atribuir a otra persona una emoción negativa a partir de una creencia equivocada.

- Codificación y puntuación. 0 puntos: no atribuye emoción negativa; 1 punto: referencia al supuesto contenido ("porque le vas a dar X"); 2 punto: razonamiento implícito ("porque no ha visto que hay Y"); 3 puntos: razonamiento mentalista explícito ("se pondrá triste porque creerá que hay X"). 


\section{LA COMPRENSIÓN DE LA PERSUASIÓN EN NIÑOS Y NIÑAS DE 6 A 12 AÑOS}

-Emoción fingida (Harris, Donnelly, Guz y Pitt-Watson, 1986; Wellman y Liu, 2004). Se evalúa la destreza para entender que una persona puede sentir una emoción, pero aparentar otra. Un personaje triste necesita aparentar lo contrario para evitar una consecuencia negativa. Se pregunta al niño qué cara pondrá (triste, neutra 0 feliz).

Codificación y puntuación. Si la cara elegida es neutra o feliz: 1 punto: referencia a la consecuencia conductual que se quiere evitar ("para que no le llamen bebé"); 2 puntos: referencia implícita a ocultar emoción ("para que no vean que está triste"); 3 puntos: justificación mentalista explícita ("para que no sepan que está triste").

- Falsa creencia de localización de segundo orden (Baron-Cohen et al., 1985; adaptada por Núñez, 1993). Evalúa la habilidad para atribuir a otro una creencia falsa sobre el conocimiento que tiene una tercera persona sobre la localización de un objeto.

Codificación y puntuación. 0 puntos: no hace un razonamiento de segundo orden; 1 punto: razonamiento de segundo orden, pero referencia implícita a los dos estados mentales ("porque no ha visto que estaba mirando"); 2 puntos: razonamiento mentalista de segundo orden con referencia explícita al primer estado mental ("porque no sabe que ha mirado"); 3 puntos: referencia explícita a los dos estados mentales ("porque se cree que no lo sabe").

Las tareas de Falsa creencia sobre contenido, Falsa creencia sobre localización de primer orden y Falsa creencia sobre localización de segundo orden corresponden a teoría de la mente cognitiva, mientras que las Tareas de creencia-emoción positiva y negativa, y de Emoción fingida son medidas de teoría de la mente afectiva.

\section{Procedimiento}

Los instrumentos fueron administrados en un aula del centro escolar durante las franjas horarias oportunas para no interrumpir el horario lectivo. En primer lugar, se aplicó la tarea de comprensión de persuasión, a continuación, se administró el test de vocabulario, y finalmente se aplicaron las tareas de teoría de la mente.

\section{RESULTADOS}

A continuación, se exponen los resultados acerca de los distintos aspectos de comprensión de la persuasión que se han analizado: en primer lugar, las inferencias acerca de la perspectiva cognitiva del hablante (creencia, motivación/actitud, intención), y en segundo lugar las inferencias acerca de la perspectiva cognitiva (creencia) y emocional (emoción) del oyente. Para dar cuenta del primer objetivo de investigación formulado, se ofrecen para cada aspecto los datos descriptivos de la distribución de las distintas categorías de análisis aplicadas. Seguidamente, para dar respuesta al segundo objetivo del estudio, se exponen para cada aspecto los resultados del análisis estadístico realizado para conocer cómo se relacionan las variables de teoría de la mente y de lenguaje con dicho aspecto. Concretamente, se han aplicado análisis de correlación de Spearman para examinar la relación entre las puntuaciones de los distintos componentes de teoría de la mente y la edad lingüística con cada uno de los aspectos de comprensión de la persuasión que se han considerado.

Perspectiva cognitiva del hablante

Tabla 1. Participantes según sus Respuestas sobre la Creencia del Hablante en la Tarea de Persuasión

\section{Perspectiva cognitiva del hablante}

\begin{tabular}{llc}
\hline \multirow{2}{*}{ Creencia } & Atribuye la creencia & $98 \%$ \\
\cline { 2 - 2 } & No atribuye la creencia & $2 \%$ \\
& \\
\hline
\end{tabular}


Como se indica en la tabla 1, casi la totalidad de los niños de la muestra atribuyen la creencia verdadera al hablante, es decir, atribuyen a Bruno que sabe que su madre le preparará comida cuando llegue a casa. No se han encontrado correlaciones estadísticamente significativas que indiquen relación entre la atribución de la creencia verdadera al hablante y la competencia mentalista y lingüística infantil.

Tabla 2. Participantes según sus Respuestas sobre la Motivación/Actitud del Hablante en la Tarea de Persuasión

\section{Perspectiva cognitiva del hablante}

Referencia al estado
mental que se pretende

\section{Motivación/Actitud}

\begin{tabular}{cc}
\hline $\begin{array}{c}\text { Referencia a } \\
\text { concecuencias } \\
\text { materiales }\end{array}$ & $80 \%$ \\
\hline No atribuye & $3 \%$
\end{tabular}

La gran mayoría de los niños evaluados (80\%) atribuye a Bruno una motivación para mentir refiriéndose a una consecuencia material (conseguir más comida), frente a una minoría de niños (17\%) que atribuye a Bruno una motivación para mentir refiriéndose al estado mental que pretende crear en el otro (que el cocinero se crea lo que el niño dice).

Tabla 3. Coeficiente de Correlación de Spearman. Relaciones de Edad Lingüística y Componentes de Comprensión de la Mente con la Motivación/Actitud del Hablante

Motivación/Actitud del Hablante

\begin{tabular}{cc}
\hline Edad lingüistica & - \\
\hline Comprensión de la Mente & .080 \\
\hline
\end{tabular}

En cuanto a la relación entre la motivación/actitud que se atribuye al hablante y la competencia mentalista y lingüística (Tabla 3), se puede afirmar que hacen referencia al estado mental que se pretende en el oyente los niños que presentan mejor ejecución en teoría de la mente, concretamente en la tarea de Falsa creencia sobre contenido (rho .174).

Tabla 4. Participantes según sus Respuestas sobre la Intención del Hablante en la Tarea de Persuasión

\begin{tabular}{ccc}
\hline Perspectiva cognitiva del hablante & \\
\hline \multirow{3}{*}{ Intención } & $\begin{array}{c}\text { Hace razonamiento de } \\
\end{array}$ & $98 \%$ \\
\cline { 2 - 3 } & $\begin{array}{c}2^{\circ} 0 \\
\text { No hace razonamiento } \\
\text { de } 2^{\circ} 0\end{array}$ & $2 \%$ \\
\hline
\end{tabular}




\section{LA COMPRENSIÓN DE LA PERSUASIÓN EN NIÑOS Y NIÑAS DE 6 A 12 AÑOS}

Como se observa en la tabla 4, casi la totalidad de los niños de la muestra revela un razonamiento de segundo orden (el niño quiere que el cocinero crea que no va a tener nada de cena cuando llegue a casa).

Tabla 5. Coeficiente de Correlación de Spearman. Relaciones de Edad Lingüística y Componentes de Comprensión de la Mente con la Intención del Hablante

\section{Intención del Hablante}

\begin{tabular}{ll}
\hline \multicolumn{1}{c}{ Edad lingüistica } & $.017^{*}$ \\
\hline Comprensión de la Mente & .076 \\
\hline${ }^{*} p<.05,{ }^{* *} p<.01$ &
\end{tabular}

En cuanto a la relación entre la intención del hablante y la competencia mentalista y lingüística se puede afirmar que atribuyen la intención al hablante realizando un razonamiento de segundo orden los niños con mayor edad lingüística (rho .237) y teoría de la mente (tarea de Emoción fingida, rho .177).

Perspectiva cognitiva y emocional del oyente

Tabla 6. Participantes según sus Respuestas sobre la Creencia y la Emoción del Oyente en la Tarea de Persuasión

\section{Perspectiva del oyente}

\begin{tabular}{|c|c|c|}
\hline \multirow{3}{*}{ Creencia } & Atribución explícita & $18 \%$ \\
\hline & $\begin{array}{l}\text { Atribución implícita } \\
T \\
\end{array}$ & $51 \%$ \\
\hline & No atribuye & $31 \%$ \\
\hline \multirow{3}{*}{ Emoción } & Atribución explícita & $26 \%$ \\
\hline & Atribución implícita & $18 \%$ \\
\hline & No atribuye & $56 \%$ \\
\hline
\end{tabular}

En la tabla 6 se refleja que el porcentaje de niños que atribuye implícitamente la creencia al oyente (51\%) es mayor que el que lo hace de manera explícita (18\%), mientras que en cuanto a la atribución de la emoción sucede al contrario, es mayor el porcentaje de niños que lo hace de forma explícita (26\%) que de forma implícita (18\%).

Tabla 7. Coeficiente de Correlación de Spearman. Relaciones de Edad Lingüística y Componentes de Comprensión de la Mente con la Creencia del Oyente

\section{Creencia del Oyente}

Edad lingüistica $\quad .089$

\section{Comprensión de la Mente}


En cuanto a la relación entre la atribución de la creencia al oyente y la competencia mentalista y lingüística, la tabla 7 refleja que los que atribuyen explícitamente la creencia del oyente (haciendo un razonamiento de segundo orden) son niños con mayor edad lingüística (rho .170).

Tabla 8. Coeficiente de Correlación de Spearman. Relaciones de Edad Lingüística y Componentes de Comprensión de la Mente con la Emoción del Oyente

\section{Emoción del Oyente}

\begin{tabular}{lc}
\hline \multicolumn{1}{c}{ Edad lingüistica } & $.003^{* *}$ \\
\hline Comprensión de la Mente & .089 \\
\hline${ }^{*}<<.05,{ }^{* *} p<.01$ &
\end{tabular}

Por último, en cuanto a la relación entre la atribución de la emoción al oyente y la competencia mentalista y lingüística, la tabla 8 refleja que los niños que atribuyen explícitamente la emoción al oyente (haciendo un razonamiento de segundo orden) son niños con mayor edad lingüística (rho .293) y teoría de la mente (concretamente la tarea de Falsa creencia de localización de segundo orden, rho .170).

\section{DISCUSIÓN}

El primer objetivo específico de este estudio consiste en analizar la capacidad de comprensión de la persuasión en los niños en función de la realización de inferencias mentalistas acerca de la perspectiva cognitiva del persuasor (su estado mental de creencia, su motivación/actitud, y su intención al intentar persuadir), así como de la realización de inferencias mentalistas acerca de la perspectiva tanto cognitiva como emocional de la persona persuadida (su estado mental de creencia y su estado emocional, ambos causados por las palabras del persuasor). El segundo objetivo específico es examinar cómo estas atribuciones que hacen los niños se relacionan con sus habilidades de teoría de la mente cognitiva y emocional y su nivel de competencia lingüística.

Es importante señalar que los estudios previos sobre comprensión de la persuasión en niños se han centrado mayoritariamente en el análisis de la perspectiva del hablante o persuasor. Concretamente, han analizado si los niños detectan que el hablante hace un uso figurado del lenguaje cuando persuade y si identifican la motivación general que tiene el hablante para decir lo que dice. Un estudio de referencia con estos dos únicos objetivos es el de Happé (1994), puesto que en su tarea de evaluación se les pregunta a los niños si es verdad lo que dice el personaje y por qué lo dice. En la tarea diseñada para el presente estudio se ha tomado como base la historia de persuasión de Happé, y se han añadido dos preguntas acerca de la perspectiva del hablante: una pregunta sobre su creencia (primer orden) y otra sobre su intención persuasiva (razonamiento de segundo orden). Además, se han incluido también preguntas acerca de la perspectiva del oyente o receptor de la persuasión, y se valora cómo atribuyen los niños la creencia y la emoción que desarrolla este como consecuencia de las palabras del persuasor. Concretamente, se valora si los niños emplean razonamientos de segundo orden de naturaleza implícita o explícita a la hora de informar acerca de lo que el oyente va a creer o de cómo se va a sentir cuando oye lo que le dice el hablante.

Tradicionalmente, el interés de la mayoría de los estudios sobre la comprensión infantil de la persuasión ha estado centrado en la evaluación de cómo los niños realizan atribuciones de estados mentales de contenido epistémico o cognitivo, y apenas se han considerado los de contenido emocional. Otra novedad destacable que presenta la tarea para la evaluación de la comprensión de la persuasión que se ha aplicado en este estudio es la consideración de los estados mentales de naturaleza afectiva o emocional. Particularmente, el análisis se ha centrado en la perspectiva del oyente, y con este objetivo se ha incluido esa pregunta con la que se valora cómo atribuyen los niños el estado emocional que desarrolla el receptor de la persuasión.

Asimismo, en este trabajo se han incluido tres tareas para la evaluación de habilidades de inferencia de estados mentales de naturaleza afectiva o emocional, adoptando una definición flexible del constructo de teoría de la 


\section{LA COMPRENSIÓN DE LA PERSUASIÓN EN NIÑOS Y NIÑAS DE 6 A 12 AÑOS}

mente (Lonigro et al., 2017). La consideración de la habilidad infantil para atribuir estados mentales emocionales en los otros ha sido interesante para esta investigación, puesto que los resultados muestran evidencia de que dicha habilidad está relacionada con los razonamientos de segundo orden que elaboran los niños a la hora de explicar la intención del hablante. Sería deseable seguir comprobando si la habilidad infantil para atribuir estados mentales emocionales se relaciona con este y otros aspectos analizados, tanto en la perspectiva del hablante como en la del oyente, en otros usos sociales del lenguaje, como la mentira o la ironía.

El análisis pormenorizado de los distintos aspectos evaluados en la tarea de comprensión de la persuasión permite extraer datos para la conclusión. En cuanto al aspecto de Creencia del hablante, no se ha encontrado que exista relación entre el reconocimiento que hacen los niños de la creencia verdadera del hablante con la competencia lingüística o mentalista infantil.

En lo que se refiere al aspecto de Motivación/Actitud del hablante, los resultados muestran evidencia de que Ios niños que hacen referencia al estado mental que se pretende en el oyente presentan mejor ejecución en teoría de la mente. Y en cuanto al aspecto de Intención del hablante, los datos obtenidos informan de que los niños que atribuyen la intención al hablante realizando un razonamiento de segundo orden presentan mayor edad lingüística y teoría de la mente.

Por otra parte, los resultados informan de que los niños que atribuyen explícitamente la creencia al oyente con un razonamiento de segundo orden son los que presentan mayor edad lingüística, y los que atribuyen explícitamente la emoción al oyente también con un razonamiento de segundo orden son niños de mayor edad lingüística y teoría de la mente.

\section{CONCLUSIONES}

En definitiva, los resultados obtenidos permiten plantear las siguientes reflexiones. Por una parte, que el nivel de desarrollo lingüístico infantil se relaciona con la inferencia de la intención del persuasor, así como con las referencias explícitas al estado de creencia y emoción que desarrolla la persona persuadida. Este hallazgo apoya la conclusión de Milligan et al. (2007) de que existe una clara relación entre el desarrollo lingüístico infantil y la adquisición de habilidades de inferencia mentalista. Los datos arrojan evidencia de que los niños que presentan mayor edad lingüística son aquellos que elaboran razonamientos mentalistas de segundo orden, los cuales requieren razonar explícitamente con los estados mentales, tanto a la hora de atribuir la intención al persuasor (el niño quiere que el cocinero crea que no va a tener nada de cena) 0 de atribuir la creencia de la persona persuadida (sí, se lo va a creer, porque no sabe que su madre le pone cena al niño).

Por otra parte, los resultados también han puesto en evidencia que las habilidades de inferencia mentalista más sofisticadas están relacionadas con la atribución de la intención al persuasor y con la referencia explícita al estado emocional de la persona persuadida. Este dato confirma que para comprender plenamente los aspectos más complejos de la persuasión, aquellos para los que se requiere atribuir estados mentales sobre estados mentales, se necesita poder realizar atribuciones mentalistas de segundo orden. Como han señalado los estudios sobre la adquisición de las habilidades mentalistas (Wellman y Liu, 2004), este logro no se alcanza antes de los 7 años en general. Este dato puede explicar que, como apuntan 0 Hare et al. (2009), la ejecución de los niños en tareas de comprensión de la persuasión presente dificultades incluso para niños de hasta 12 años de edad.

\section{FINANCIACIÓN}

Este trabajo ha sido financiado por el Plan Propio de Investigación de la Universidad de Málaga. Programa para la promoción de proyectos de investigación en Ciencias Sociales, Código: 2019_1. 


\section{REFERENCIAS BIBLIOGRÁFICAS}

Baron-Cohen, S., Leslie, A.M., y Frith, U. (1985). Does the autistic child have a "theory of mind"? Cognition, 21, $37-46$.

Bartsch, K., London, K. y Campbell, M.D. (2007). Children`s attention to beliefs in interactive persuasion tasks. Developmental Psychology, 43, 111-120.

Donohue, T., Henke, L. y Donohue, W. (1980). Do kids know what TV commercials intend? Journal of Advertising Research, 20, 51-57.

Dunn, LI.M., Dunn, L.M. y Arribas, D. (2006). Test de Vocabulario en Imágenes Peabody. TEA Ediciones.

Happé, F. G. (1994). An advanced test of theory of mind: Understanding of story characters' thoughts and feelings by able autistic, mentally handicapped, and normal children and adults. Journal of autism and Developmental disorders, 24(2), 129-154

Harris, P.L., Donnelly, K., Guz, G.R. y Pitt-Watson, R. (1986). Children s understanding of the distinction between real and apparent emotion. Child Development, 57, 895-909.

Harris, P.L., Johnson, C.N., Hutton, D., Andrews, G. y Cooke, T. (1989). Young children s theory of mind and emotion. Cognition and Emotion, 3, 379-400.

Jenkins, J.M. y Astington, J.W. (1996). Cognitive factors and family structure associated with theory of mind development in young children. Developmental Psychology, 31(1), 70-78.

Lapierre, M.A. (2015). Development and persuasion understanding: predicting knowledge of persuasion/selling intent from children s theory of mind. Journal of Communication, 65, 423-442.

Lillard, A.S. y Kavanaugh, R.D. (2012). The contribution of symbolic skills to the development of an explicit theory of mind: scale models, language, and pretend play.

Lonigro, A., Baiocco, R., Baumgartner, E. y Laghi, F. (2017). Theory of mind, affective empathy, and persuasive strategies in school-aged children. Infant and Child Development, 26(6), e2022.

McAlister, A.R. y Cornwell, T.B. (2009). Preschool children s persuasion knowledge: the contribution of theory of mind. Journal of Public Policy and Marketing, 28(2), 175-185.

Miller, S.A. (2012). Theory of mind. Beyond the preschool years. Psychology Press.

Milligan, K., Astington, J.W. y Dack, L.A. (2007). Language and theory of mind: meta-analysis of the relation between language ability and false-belief understanding. Child Development, 78(2), 622-646.

Núñez, M. (1993). Teoría de la mente: Metarrepresentación, creencias falsas y engaño en el desarrollo de una psicología natural. Tesis doctoral sin publicar. Universidad Autónoma de Madrid.

0 Hare, A.E., Bremner, L., Nash, M., Happé, F.G.E. y Pettigrew, L.M. (2009). A clinical assessment tool for advanced theory of mind performance in 5 to 12 year olds. Journal of Autism and Developmental Disorders, 39(6), 916-928.

Perner, J., Leekam, S. y Wimmer, H. (1987). Three-year olds difficulty with false belief: The case for a conceptual deficit. British Journal of Developmental Psychology, 5, 125-137.

Premack, D. y Woodruff, G. (1978). Does the chimpanzee have a theory of mind? Behavioral and Brain Sciences, 1(4), 515-526.

Watson, A.C., Painter, K.M. y Bornstein, M.H. (2001). Longitudinal relations between 2-year-olds theory of mind. Journal of Cognition and Development, 2(4), 449-457.

Wellman, H., Cross, D. y Watson, J. (2001). Meta-analysis of theory-of-mind development: The truth about falsa belief. Child Development, 72(3), 655-684.

Wellman, H. y Liu, D. (2004). Scaling of Theory-of-Mind tasks. Child Development, 75(2), 523-541.

Wimmer, H. y Perner, J. (1983). Beliefs about beliefs: representation and constraining function of wrong beliefs in young children s understanding of deception. Cognition, 13(1), 103-128. 
